\title{
Estimating a Dynamic Effect of Soda Intake on Pediatric Dental Caries Using Targeted Maximum Likelihood Estimation Method
}

\author{
Sungwoo Lim Marisol Tellez Amid I. Ismail \\ Department of Pediatric Dentistry and Community Oral Health Sciences Kornberg School of Dentistry, \\ Philadelphia, PA, USA
}

\section{Keywords}

Biostatistics · Epidemiology · Dental caries

\begin{abstract}
An effect of soda intake on dental caries in young children (birth to 5 years) may vary over time. Estimating a dynamic effect may be challenging due to time-varying confounding and loss to follow-up. The purpose of this paper is to demonstrate utility of targeted maximum likelihood estimation (TMLE) method in addressing longitudinal data analysis challenges and estimating a dynamic effect of soda intake on pediatric caries. Data came from the Detroit Dental Health Project, a 4-year cohort study of low-income African-American children and caregivers. The sample included 995 child-caregiver pairs who participated in 200203 (W1) and were followed up in 2004-05 (W2) and 2007 (W3). The outcome was counts of caries surfaces at W3, and the exposure was child's soda intake at W1 and W2. Timevarying covariates included caregiver's smoking status, oral health fatalism, and social support. Forty-three percent of children consistently consumed soda at W1 and W2, whereas $21 \%$ were nonconsumers throughout 2 surveys. The remaining 35\% switched intake status between W1 and W2.
\end{abstract}

Association between soda intake patterns and caries was tested using TMLE. Children with a consistent soda intake had 1.03 more caries lesions at W3 than those with consistently no soda intake $(95 \% \mathrm{Cl} 0.09-1.97)$ on average. If soda was consumed only at W1 or W2, an estimated effect of soda on caries development at W3 was no longer statistically significant. In conclusion, consistent soda intake during the early childhood led to one additional caries tooth surface. The study highlights utility of TMLE in pediatric caries research as it can handle modeling challenges associated with longitudinal data.

(c) 2019 S. Karger AG, Basel

\section{Introduction}

Soda intake is one of the main risk factors for dental caries among children [Lim et al., 2008; Ismail et al., 2009]. As children grow and become more independent, trajectories of their dietary behaviors and dental caries can rapidly change [Anzman et al., 2010; Ismail et al., 2015]. Yet, little is known about the extent to which this association varies over time during childhood. It may be attributed to challenges in measuring a dynamic effect of

\section{KARGER}

(c) 2019 S. Karger AG, Basel

E-Mail karger@karger.com

www.karger.com/cre
Marisol Tellez

Pediatric Dentistry and Community Oral Health Sciences Kornberg School of Dentistry Temple University

3223 N. Broad Street, Suite L218, Philadelphia, PA 19140 (USA)

E-Mail marisol.tellez.merchan@ temple.edu 
soda intake on dental caries. Drawing inference from longitudinal, time-varying data requires special statistical techniques and statistical assumptions. Recent studies show that targeted maximum likelihood estimation (TMLE) method can have a better performance in terms of addressing bias due to time-varying confounding and drawing causal inference as opposed to conventional regression analysis [Van der Laan, 2010; Petersen et al., 2014]. It is because an estimator from the conventional regression method is unbiased under the assumption that a treatment effect is homogeneous across all levels of confounders, which is highly untenable when confounding is time varying [Keil et al., 2014]. Evidence also suggests that TMLE outperforms other causal estimation methods such as G-computation and propensity score methods [Schuler and Rose, 2017; Luque-Fernandez et al., 2018]. A causal estimator from TMLE, as opposed to G-computation, is more efficient because TMLE has an additional estimation process to optimize a parameter of interest (e.g., average treatment effect, risk difference) [Schuler and Rose, 2017]. Compared with propensity score method, TMLE has greater potential to produce an unbiased estimator because its substitution method is less affected by outliers or data sparsity [Schuler and Rose, 2017]. Further, estimation of both outcome and exposure using covariates, which is a unique property of TMLE known as a double robust approach, increases a chance to reduce bias due to model misspecification [Schuler and Rose, 2017].

The objective of this study is to demonstrate the utility of TMLE in dental caries research. Specifically, we used both TMLE and regression analysis to test and quantify association between a particular soda intake pattern and dental caries over 4 years among low-income African-American children.

\section{Materials and Methods}

Data for this study were obtained from the Detroit Dental Health Project, a longitudinal cohort study that was designed to investigate the oral health determinants of low-income AfricanAmerican children and their caregivers. The sample was selected using a stratified 2-stage area probability sample of households in Detroit, Michigan. The eligible households comprised of at least one caregiver of a child ages $0-5$ years. Details of the sampling and data collection procedures have been described in previous reports [Ismail et al., 2008]. Participants ( $n=1,021$ child-caregiver pairs) at baseline (W1) were followed up in 2004-05 (W2) and 2007 (W3). In this study, we focused on 995 pairs of child and caregivers who reported their soda intake. Of these, $229(23 \%)$ and 360 (36\%) were lost to follow-up in W2 and W3 surveys, respectively.
The study protocol was approved by the Institutional Review Board for Health Sciences at the University of Michigan, and caregivers of all participants gave written consent for inclusion in this study (IRB\# 1325).

\section{Variables}

The study outcome was counts of caries surfaces at W3 in both primary and permanent dentitions. As described in the previous report [Ismail et al., 2008; Ismail et al., 2015], 4 dentists assessed the baseline caries status of all tooth surfaces using the International Caries Detection and Assessment System. A new team of trained dentists (except for one baseline examiner and the trainers [gold standard]) conducted the examinations at follow-up. Before dental caries assessment, all teeth were cleaned and dried. Training of examiners started with a 2 -week intensive review and repeated examinations followed by reliability assessments and reviews of findings throughout each data collection cycle. The "gold standard" was the same examiner throughout the study. Intra-examiner kappa coefficients at baseline were between 0.57 (for 1 examiner) and $0.77-0.78$ (for the other 3 examiners) when initial caries lesions were classified as a distinct category within a sound to extensive caries scale. Inter-examiner kappa coefficients ranged between 0.42 (for 1 examiner) and $0.65-0.79$ (for 3 examiners). For the follow-up examination, the intra- and inter-examiner kappa coefficients were above 0.70 . Since prevalent caries was a strong predictor of caries incidence in previous studies, we also included counts of caries surfaces at W1 and W2 as intermediary variables in this study [Ismail et al., 2009; Lim et al., 2015]. The exposure variable was child's carbonated sugary drink (soda) intake at W1 and W2. Using Block's Kids Food Frequency Questionnaire, we collected child's weekly frequencies of soda intake during the preceding week from caregivers and grouped responses into 2 categories (yes, no).

In addition to the outcome and exposure variables, we included covariates that were identified as a common cause of children's caries and soda intake according to a direct acyclic graphic (Appendix 1). Empirically, some of these variables (e.g., age, baseline caries, current smoking, oral health fatalism) were also significant risk factors of dental caries in the previous studies of the same samples of children [Ismail et al., 2009; Lim et al., 2015]. Time-varying covariates included caregiver's smoking status, oral health fatalism, and social support at W1 and W2. Caregivers who reported smoking $\geq 100$ cigarettes in their life and currently smoke were defined as current smokers, whereas those who have never smoked or smoked $<100$ cigarettes in their life were considered as noncurrent smokers. Oral health fatalism was measured by asking the caregivers whether they agree or disagree with a statement that "most children eventually develop dental cavities." The answers were grouped "high" (neutral, agreed, and strongly agreed) and "low" (disagree and strongly disagree). Caregivers who responded "yes" to all 5 questions (help with errands, help with financial needs, help with childcare needs, help with transportation needs, and availability for emotional support) were identified as those with social support. Lastly, time-invariant covariates from W1 data included age (child and caregiver), sex (child), caries (child), perceived racial discrimination (caregiver), household size, depressive symptoms (caregiver), household income, education (caregiver), and histories of residential movement in the past 5 years. 
Statistical Analysis

We drew causal inference for longitudinal data using TMLE. Causation is established when outcome under the actual exposure (e.g., soda intake) is different from outcome under the counterfactual condition (e.g., no soda intake) in a same individual. Counterfactual outcomes (e.g., caries for a soda-drinking child under the hypothetical condition that soda was not consumed) are unobserved and in TMLE these are considered missing data and substituted with predicted values [Van der Laan, 2010]. Specifically, outcome model for dental caries was constructed using time-invariant covariates (child's age at W1, child's sex, caregiver's age at W1, caregiver's depressive symptoms at W1, caregiver's perceived racial discrimination at $\mathrm{W} 1$, caregiver's education at $\mathrm{W} 1$, household income at $\mathrm{W} 1$, household size at $\mathrm{W} 1$, histories of residential movement in the past 5 years), histories of outcomes (caries at W1, caries at W2), exposure (soda intake at W1, soda intake at W2), and time-varying covariates (caregiver's smoking at W1 and W2, caregiver's oral health fatalism at W1 and W2, caregiver's social support at W1 and W2), which in turn produced conditional expectations of dental caries under the actual soda intake condition as well as counterfactuals for each child. Two additional models for soda intake (propensity score) and loss to follow-up (censoring) were constructed using the same set of covariates from the outcome model. Likelihood of receiving an exposure (propensity score; soda vs. no soda) and likelihood of being lost to follow-up (censoring) were estimated and then incorporated into predicted values of the outcome. Starting from the last time point of the follow-up, this process repeated at each time point in a backward order. Theoretical and empirical studies show that this recursive conditional likelihood efficiently controls for bias due to time-varying confounding and addresses positivity (i.e., sufficient data for both actual soda intake and counterfactual in each level of confounders) and identifiability issues [Bang and Robins, 2005; Petersen et al., 2014]. Use of the same set of covariates in outcome, propensity score, and censoring models (known as doubly robust property) also helps reduce bias due to model misspecification [Petersen et al., 2014]. Lastly, updated conditional expectations by exposure status via recursive conditional likelihood were compared and averaged over all study children, yielding a causal estimand (= difference between the observed and counterfactual outcome). Inference was made by influence curves.

TMLE was estimated under 3 different scenarios (scenario \#1: W1 soda intake - W2 soda intake, scenario \#2: W1 no soda intake - W2 soda intake, scenario \#3: W1 soda intake - W2 no soda intake) against a counterfactual pattern (W1 no soda intake - W2 no soda intake). Estimation was made using both parametric regression and machine learning approach. Specifically, the latter uses a data adaptive algorithm where a series of estimators were calculated via various methods such as random forest, elastic net, regression trees, and generalized additive modeling, and the best weighted combination of estimators are selected via cross-validation, which could address bias due to model misspecification and other violations of statistical assumptions.

As a sensitivity analysis, we estimated difference in dental caries at W3 by soda intake via 2 conventional Poisson regression models. While both models had time-invariant covariates from W1 survey, 2 models differed by inclusion of time-varying covariates (the first model: time-varying covariates at W2, the second model: time-varying covariates at W1 and W2).
Table 1. Selected baseline characteristics of caregivers and children

\section{$n(\%)$}

Income

Less than USD 10,000

More than USD 10,000

$437(44)$

Education

Less than high school

High school diploma

$555(56)$

Some college or more

$456(46)$

$314(32)$

$222(22)$

Has moved in the past 5 years

$768(77)$

Frequent discrimination

Never

$330(33)$

$<5$ areas

$459(46)$

$\geq 5$ areas

$203(20)$

Current smoking

$418(42)$

Social support

$646(65)$

Oral health fatalistic belief

$761(77)$

Gender of caregiver

Male

Female

Caregiver's average age, mean $\pm \mathrm{SE}$

$29.1 \pm 0.3$

Gender of child

Male

$496(50)$

Female

$496(50)$

Child's average age, mean \pm SE $\quad 2.7 \pm 0.1$

Child's average dental caries, mean \pm SE $\quad 2.1 \pm 0.2$

In TMLE analysis, estimates were weighted using baseline weights because likelihood of lost to follow-up in W2 and W3 (i.e., censoring) was explicitly modeled and estimated using time-varying and time-invariant confounders. In regression analysis, the design-based approach was used to account for complex sample design. Statistical analyses were conducted using $\mathrm{R}$ software LTMLE package (for TMLE analysis) and survey package (for regression analysis) [Lendle et al., 2017; Lumley, 2004]. Statistical significance was determined by 2 -sided $p$ value $<0.05$.

\section{Results}

Forty-four percent of caregivers had their annual household incomes lower than USD 10,000, while 46\% did not have a high school degree (Table 1). Ninety-six percent of caregivers were female, and their average age was 29 years. Seventy-seven percent reported to move at least once in the past 5 years, and approximately $75 \%$ of caregivers reported frequent discrimination in daily lives. Forty-two percent were current smokers and $65 \%$ reported to receive either emotional support or help with one of 4 needs (errand, childcare, transportation, and finance). A half of the children were female, 
Table 2. Selected characteristics and dental caries by children's soda intake patterns

\begin{tabular}{|c|c|c|c|c|c|}
\hline & \multicolumn{4}{|c|}{ W1-W2 soda intake pattern } & \multirow[t]{2}{*}{$p$ value } \\
\hline & $\begin{array}{l}\text { W1 no soda - } \\
\text { W2 no soda }\end{array}$ & $\begin{array}{l}\text { W1 no soda - } \\
\text { W2 soda }\end{array}$ & $\begin{array}{l}\text { W1 soda - } \\
\text { W2 no soda }\end{array}$ & $\begin{array}{l}\text { W1 soda - } \\
\text { W2 soda }\end{array}$ & \\
\hline Number & 125 & 118 & 91 & 248 & \\
\hline \multicolumn{6}{|l|}{ Income } \\
\hline Less than USD 10,000 & 40 & 49 & 40 & 47 & \multirow[t]{2}{*}{0.42} \\
\hline More than USD 10,000 & 60 & 51 & 60 & 53 & \\
\hline \multicolumn{6}{|l|}{ Education } \\
\hline Less than high school & 42 & 46 & 49 & 48 & \multirow[t]{3}{*}{0.50} \\
\hline High school diploma & 29 & 38 & 35 & 30 & \\
\hline Some college or more & 29 & 16 & 16 & 22 & \\
\hline Has moved in the past 5 years & 77 & 69 & 70 & 80 & 0.11 \\
\hline \multicolumn{6}{|l|}{ Frequent discrimination } \\
\hline Never & 41 & 41 & 32 & 28 & \multirow[t]{3}{*}{0.35} \\
\hline$<5$ areas & 38 & 38 & 51 & 50 & \\
\hline$\geq 5$ areas & 21 & 21 & 17 & 22 & \\
\hline \multicolumn{6}{|l|}{ Smoking at } \\
\hline $\mathrm{W} 1$ & 28 & 40 & 41 & 47 & 0.03 \\
\hline $\mathrm{W} 2$ & 27 & 41 & 36 & 45 & 0.02 \\
\hline \multicolumn{6}{|l|}{ Oral health fatalistic belief at } \\
\hline $\mathrm{W} 1$ & 70 & 80 & 75 & 78 & 0.35 \\
\hline W2 & 65 & 67 & 74 & 77 & 0.10 \\
\hline \multicolumn{6}{|l|}{ Social support at } \\
\hline W1 & 68 & 80 & 62 & 63 & 0.06 \\
\hline W2 & 63 & 67 & 61 & 70 & 0.56 \\
\hline Caregiver's average age (SE) & $27.7(0.8)$ & $28.5(0.6)$ & $29.1(1.0)$ & $30.1(0.6)$ & 0.29 \\
\hline Child's average age (SE) & $2.3(0.2)$ & $1.7(0.2)$ & $3.1(0.2)$ & $3.1(0.1)$ & $<0.001$ \\
\hline \multicolumn{6}{|l|}{ Child's average dental caries at } \\
\hline W1 (SE) & $1.3(0.5)$ & $0.9(0.4)$ & $2.4(0.7)$ & $2.5(0.4)$ & $<0.001$ \\
\hline W2 (SE) & $1.6(0.5)$ & $2.1(0.4)$ & $2.5(0.5)$ & $3.7(0.4)$ & $<0.001$ \\
\hline W3 (SE) & $2.2(0.5)$ & $2.9(0.6)$ & $3.0(0.5)$ & $3.8(0.4)$ & 0.17 \\
\hline
\end{tabular}

Figures are percentages unless indicated otherwise.

W1, Wave 1, baseline; W2, Wave 2, 1st follow-up; W3, Wave 3, 2nd follow-up.

and their average age was 3 years. At baseline, average carious tooth surfaces among children were 2.1 (SE $0.2)$.

Among noncensored samples (i.e., participants of all 3 waves), $43 \%$ of children reported to consume soda at both W1 and W2, whereas 21\% were nonconsumers throughout W1 and W2. Twenty percent switched from nonconsumers at $\mathrm{W} 1$ to consumers at $\mathrm{W} 2$, and remaining $15 \%$ switched from consumers to nonconsumers. Children with consistent soda intake were much older and had a greater number of tooth surfaces with carious lesions (Table 2). Caregivers' characteristics were quite similar regardless of children's soda intake patterns. One exception was that caregivers of children with consistent soda intake were more likely to be smokers.

Dynamic Effect of Soda on Pediatric Caries
Figure 1 shows that distributions of caries were rightskewed across different age groups of children who were participating in all 3 waves. As children got older over 4 -year examinations, a bar around zero caries decreased, indicating that more children experienced caries from W1 through W3. However, the overall shape of the distributions remained similar across 3 time points.

Table 3 shows the results from TMLE. Accounting for time-varying and time-invariant confounding, children with a consistent soda intake pattern developed 3.41 caries surfaces at W3 (95\% CI 2.84-3.98) on average, whereas those with consistently no soda intake developed 2.38 caries surfaces (95\% CI 1.61-3.14). Comparing these 2 estimates, TMLE analysis determined a significant additive effect of a consistent soda intake at 


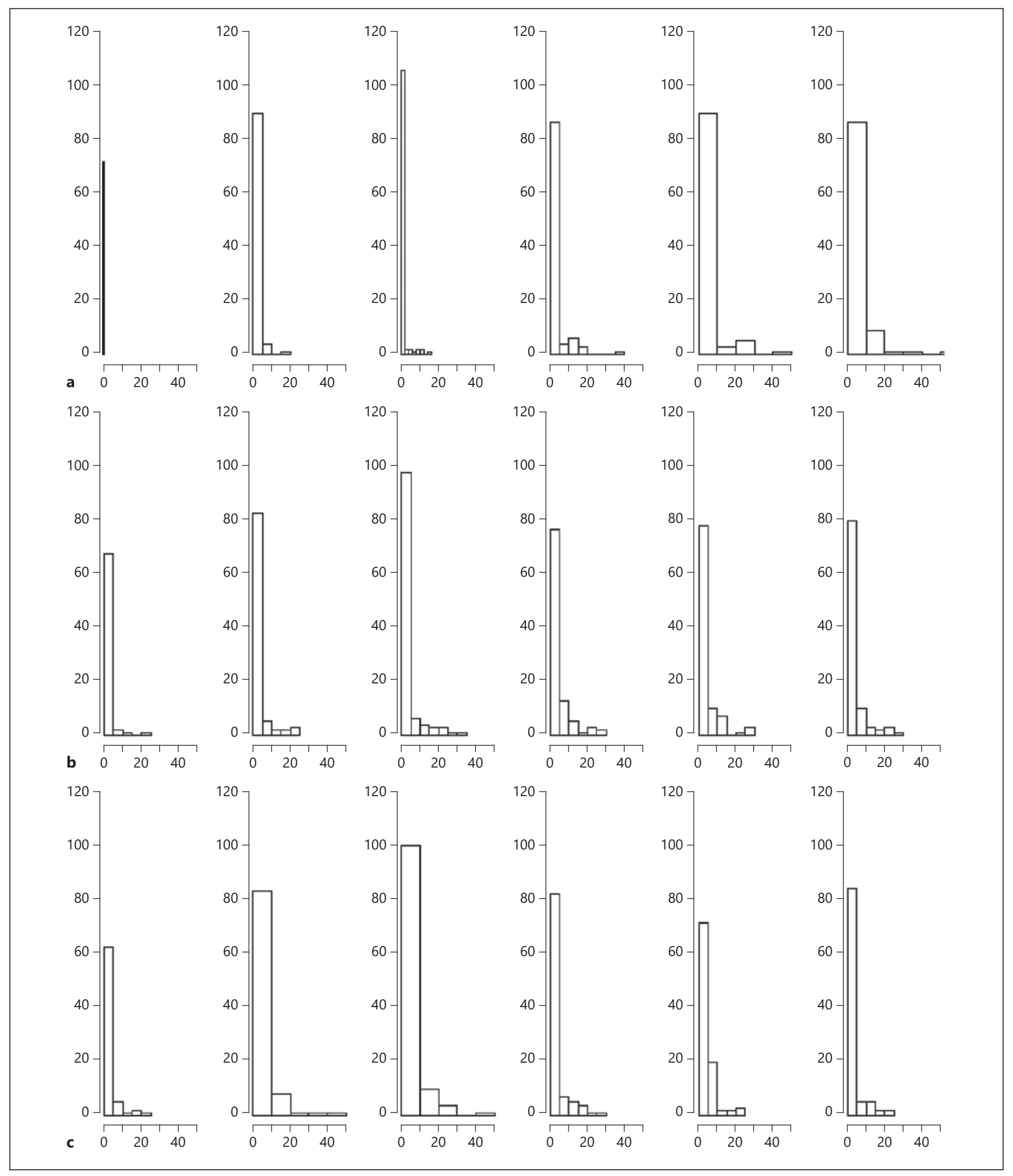

Fig. 1. Histograms of dental caries by children's baseline age. Caries at W1 (a); Caries at W2 (b); Caries at W3 (c). W1, Wave 1, baseline; W2, Wave 2, 1st follow-up; W3, Wave3, 2nd follow-up. 
Table 3. Results from targeted maximum likelihood estimation

\begin{tabular}{lllll}
\hline & Estimates & SE & 95\% CI & $p$ value \\
\hline Scenario \#1: W1 soda - W2 soda & 3.41 & 0.28 & 2.84 to 3.98 & $<0.001$ \\
Scenario \#2: W1 no soda - W2 soda & 3.18 & 0.29 & 2.61 to 3.74 & $<0.001$ \\
Scenario \#3: W1 soda - W2 no soda & 3.14 & 0.23 & 2.70 to 3.59 & $<0.001$ \\
Counterfactual: W1 no soda - W2 no soda & 2.38 & 0.39 & 1.61 to 3.14 & $<0.001$ \\
\hline Scenario \#1 vs. counterfactual & 1.03 & 0.48 & 0.09 to 1.97 & 0.03 \\
Scenario \#2 vs. counterfactual & 0.75 & 0.42 & -0.08 to 1.58 & 0.08 \\
Scenario \#3 vs. counterfactual & 0.73 & 0.40 & -0.06 to 1.53 & 0.07 \\
\hline
\end{tabular}

W1, Wave 1, baseline; W2, Wave 2; estimates are mean counts of caries lesions at W3 (2nd follow-up).

Table 4. Results from the regression analyses

\begin{tabular}{|c|c|c|c|c|}
\hline & \multicolumn{2}{|c|}{ Model 1} & \multicolumn{2}{|c|}{ Model 2} \\
\hline & $\mathrm{RR}$ & $95 \% \mathrm{CI}$ & $\mathrm{RR}$ & $95 \% \mathrm{CI}$ \\
\hline Child's age & 0.96 & $0.87-1.06$ & 0.93 & $0.83-1.05$ \\
\hline Caregiver's age & 1.01 & $0.99-1.03$ & 1.01 & $1.00-1.02$ \\
\hline Smoking at W1 & NA & & 0.94 & $0.64-1.38$ \\
\hline Smoking at W2 & 1.12 & $0.84-1.48$ & 1.10 & $0.80-1.50$ \\
\hline Frequent discrimination & 0.94 & $0.78-1.15$ & 0.87 & $0.73-1.04$ \\
\hline Social support at W1 & NA & & 0.84 & $0.63-1.11$ \\
\hline Social support at W2 & 0.82 & $0.63-1.07$ & 0.72 & $0.53-0.97$ \\
\hline Oral health fatalistic belief at W1 & NA & & 1.22 & $0.75-2.00$ \\
\hline Oral health fatalistic belief at W2 & 1.06 & $0.75-1.50$ & 1.03 & $0.76-1.39$ \\
\hline Has moved in the past 5 years & 1.02 & $0.72-1.47$ & 1.05 & $0.75-1.46$ \\
\hline Soda intake at $\mathrm{W} 1$ & NA & & 1.25 & $0.85-1.84$ \\
\hline Soda intake at W2 & 1.33 & $0.98-1.82$ & 1.12 & $0.84-1.50$ \\
\hline \multicolumn{5}{|l|}{ Household income: } \\
\hline More than USD 10,000 vs. less than USD 10,000 & 1.10 & $0.80-1.51$ & 1.12 & $0.86-1.46$ \\
\hline \multicolumn{5}{|l|}{ Education } \\
\hline High school diploma vs. less than high school & 1.18 & $0.93-1.49$ & 1.11 & $0.87-1.40$ \\
\hline \multicolumn{5}{|l|}{ Education } \\
\hline Some college or more vs. less than high school & 0.95 & $0.64-1.41$ & 0.98 & $0.69-1.41$ \\
\hline Dental caries at W1 & 1.04 & $1.02-1.06$ & 0.99 & $0.96-1.01$ \\
\hline Dental caries at W2 & NA & & 1.09 & $1.07-1.11$ \\
\hline
\end{tabular}

W1, Wave 1, baseline; W2, Wave 2, 1st follow-up; W3, Wave 3, 2nd follow-up; RR stands for relative risk for caries at W3.

W1 and W2 (scenario \#1) versus no intake (1.03, 95\% CI 0.09-1.97). In other words, a consistent soda intake over 2 years, as opposed to a consistent no soda intake, led to one more caries surfaces at the 4 th years postbaseline. However, if soda was consumed only at W1 or W2 (i.e., scenario \#2 and \#3), an estimated effect of soda on caries development at W3 was not statistically significant.

Dynamic Effect of Soda on Pediatric Caries
According to the sensitivity analysis, soda intake at W2 was positively associated with caries at $\mathrm{W} 3$, but this association was not statistically significant (RR 1.33, 95\% CI $0.98-1.82$ ). In an additional sensitivity analysis where both $\mathrm{W} 1$ and $\mathrm{W} 2$ predictors were included in the regression model, a positive association between soda intake at both W1 and W2 and caries was observed, but not statistically significant (Table 4 ). 


\section{Discussion}

In this study, we found that consistent soda intake at baseline and 2-year follow-up was associated with about 1 additional caries tooth surface at the 4 th year after baseline among African-American children. Inconsistent soda intake (e.g., early consumer or late consumer), as opposed to no soda intake, was not associated with developing new dental caries in these study children. To estimate and test causal associations, we employed TMLE method to address violations of causal assumptions, including time-varying confounding and model misspecification.

The traditional regression analyses showed a positive association between soda intake and dental caries, but it was not statistically significant. Difference between TMLE and regression analyses results may be due to difference in their approaches to perform estimation. First, TMLE uses the whole sample to estimate observed and counterfactual outcomes. Regardless of actual exposure status (e.g., soda vs. no soda), this method can estimate outcome $(=\mathrm{d} 2$ at W3) under counterfactual exposure status for the same child. On the other hand, regression approach can only estimate observed outcomes, which limits its ability to establish exchangeability and make causal inference. Second, TMLE employs a nonparametric model and doubly robust estimating. Specifically, TMLE uses a machine learning technique for model building and consistently uses the same set of covariates to estimate outcome, propensity of soda intake, and loss to follow-up. These analytic techniques can address bias due to model misspecification, compared with regression approach. Third, TMLE can account for time-varying confounding and allow for making causal inference under different treatment scenarios (e.g., soda - soda vs. no soda - no soda). Since the regression approach is only based on observations, it does not have the capacity to test various treatment patterns.

The current finding also demonstrates that TMLE is more advantageous than the extended cox model, which is an alternative modeling approach to handle time-varying data [Ghazal et al., 2018]. Although the extended cox model can explicitly incorporate time-varying property, it requires a large sample size to account for confounding and only handles time to event outcome [Ghazal et al., 2018]. Especially when data are collected via observational studies, confounding can substantially bias estimates, which can undermine a benefit from handling time-varying data.

Although the study clearly shows advantages of TMLE over conventional regression methods, some limitations should be noted. First, soda intake was measured using a binary "yes" versus "no" indicator. Risk of caries is likely to be much higher among children who have consumed soda 3-4 times versus 1-2 times per week, but gradient of risk by a frequency of intake cannot be estimated because a current TMLE algorithm only accepts a binary exposure variable. Second, although various sets of child- and caregiver-level covariates were included to account for confounding, bias in estimates due to unobserved confounding cannot be ruled out. Lastly, data other than caries came from selfreports and could be biased due to social desirability or differential recall.

In conclusion, if researchers aim to test whether a particular pattern of treatment predicts an outcome using observational data, we believe that TMLE is more appropriate than the conventional regression approach where time-varying confounding and bias due to misspecification may not be addressed.

\section{Acknowledgments}

This study was supported with funding from the National Institute on Dental and Craniofacial Research (NIDCR) grant \# U-54 DE 14261-01, the Delta Dental Fund of Michigan, and the University of Michigan's Office of Vice President for Research.

\section{Statement of Ethics}

The study protocol was approved by the Institutional Review Board for Health Sciences at the University of Michigan, and caregivers of all participants gave written consent for inclusion in this study (IRB\# 1325).

\section{Disclosure Statement}

Amid I Ismail cofounded the International Caries and Assessment System. He also serves in the Board of Directors at SS White Inc.

\section{Author Contributions}

All authors assume responsibility for the results reported herein. A.I. conducted the primary data collection. A.I., S.L., and M.T. were involved in the conceptualization, analysis, and interpretation and read and approved the submission of this manuscript 


\section{Appendix 1}

Relationships Among Outcome, Exposure, and Covariates by a Directed Acyclic Graph

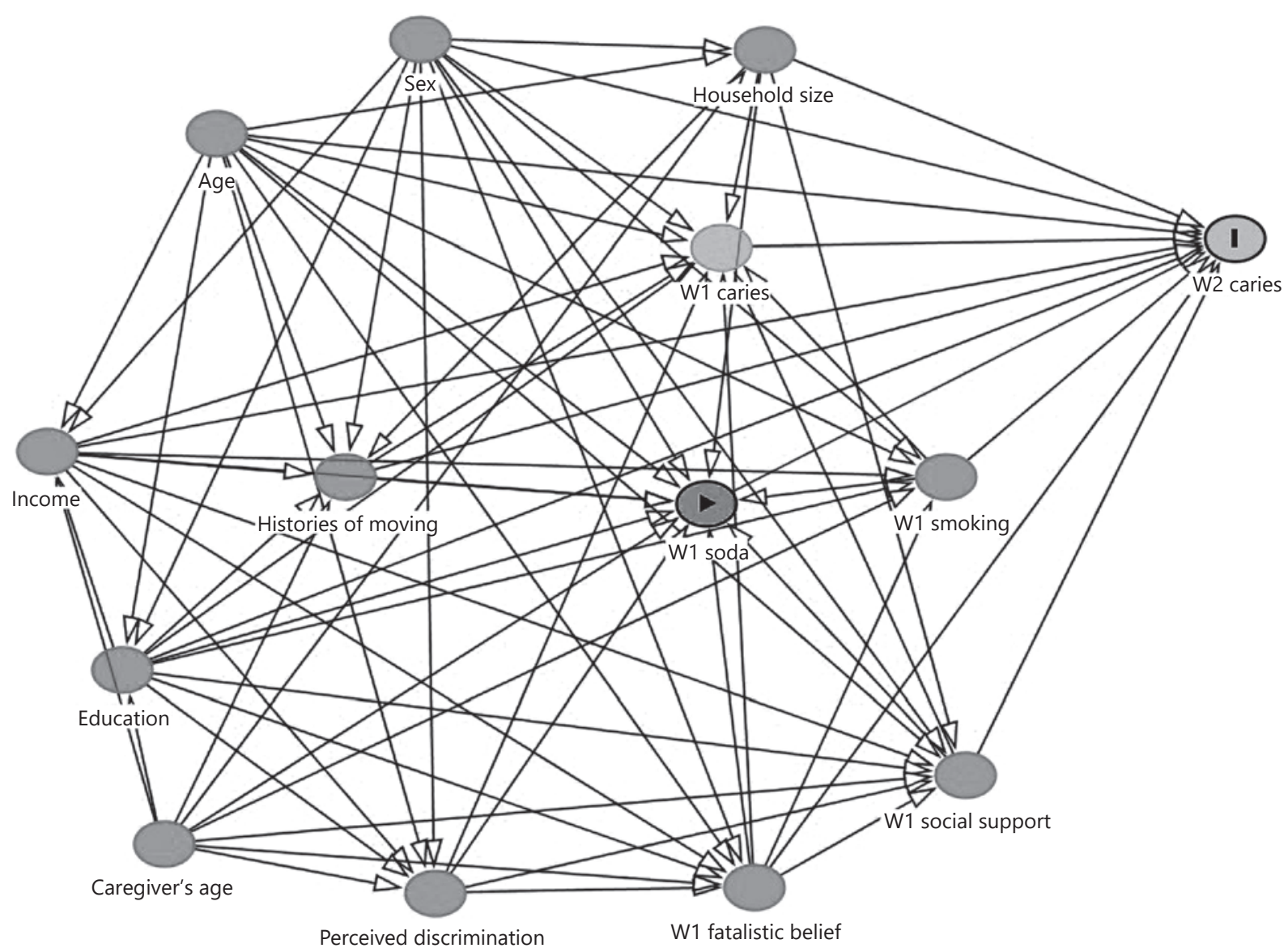

W1, Wave 1, baseline; W2, Wave 2, 1st follow-up; W3, Wave 3, 2nd follow-up; the relationships among W1 and W2 variables, which are described above, can be extended to W2 and W3 variables; variables with red color are identified as a common cause of soda intake and caries, confounding a pathway from exposure to outcome.

\section{References}

Anzman SL, Rollins BY, Birch LL. Parental influence on children's early eating environments and obesity risk: implications for prevention. Int J Obes. 2010 Jul;34(7):1116-24.

Bang H, Robins JM. Doubly robust estimation in missing data and causal inference models. Biometrics. 2005 Dec;61(4):962-73.

Ghazal TS, Levy SM, Childers NK, Carter KD, Caplan DJ, Warren JJ, et al. Mutans Streptococci and Dental Caries: A New Statistical Modeling Approach. Caries Res. 2018;52(3):246-52.

Ismail AI, Lim S, Tellez M. Tooth surface level caries progression in the primary dentition among preschool children. Caries Res. 2015; 49(4):442-8.
Ismail AI, Sohn W, Lim S, Willem JM. Predictors of dental caries progression in primary teeth. J Dent Res. 2009 Mar;88(3):270-5.

Ismail AI, Sohn W, Tellez M, Willem JM, Betz J, Lepkowski J. Risk indicators for dental caries using the International Caries Detection and Assessment System (ICDAS). Community Dent Oral Epidemiol. 2008 Feb;36(1):55-68.

Keil AP, Edwards JK, Richardson DB, Naimi AI, Cole SR. The parametric g-formula for timeto-event data: intuition and a worked example. Epidemiology. 2014 Nov;25(6):889-97.

Lendle S, Schwab J, Petersen ML, van der Laan MJ. Ltmle: an R package implementing tar- geted minimum loss-based estimation for longitudinal data. J Stat Softw. 2017;81(1).

Lim S, Sohn W, Burt BA, Sandretto AM, Kolker JL, Marshall TA, et al. Cariogenicity of soft drinks, milk and fruit juice in low-income african-american children: a longitudinal study. J Am Dent Assoc. 2008 Jul;139(7):959_ 67.

Lim S, Tellez M, Ismail AI. Dental caries development among African American children: results from a 4-year longitudinal study. Community Dent Oral Epidemiol. 2015 Jun;43(3): 200-7.

Lumley T. Analysis of complex survey samples. J Stat Softw. 2004;9(1):1-9. 
Luque-Fernandez MA, Belot A, Valeri L, Cerulli G, Maringe C, Rachet B. Data-adaptive estimation for double-robust methods in populationbased cancer epidemiology: risk differences for lung cancer mortality by emergency presentation. Am J Epidemiol. 2018 Apr;187(4):871-8.
Petersen M, Schwab J, Gruber S, Blaser N, Schomaker M, van der Laan M. Targeted maximum likelihood estimation for dynamic and static longitudinal marginal structural working models. J Causal Inference. 2014 Jun;2(2): $147-85$.

Schuler MS, Rose S. Targeted maximum likelihood estimation for causal inference in observational studies. Am J Epidemiol. 2017 Jan; 185(1):65-73.
Sheiham A, James WP. Diet and dental caries: the pivotal role of free sugars reemphasized. J Dent Res. 2015 Oct;94(10):1341-7.

van der Laan MJ. Targeted maximum likelihood based causal inference: Part I. Int J Biostat. 2010 Jan;6(2):2. 